\title{
Psychological correlates of nonspecific electrodermal responses
}

\author{
Dindar S. Bari \\ Department of Physics, University of Zakho, Zakho, Kurdistan region, Iraq \\ E-mail any correspondence to: dindar.bari@uoz.edu.krd
}

\begin{abstract}
Spontaneous fluctuations in electrodermal responses are known as nonspecific electrodermal responses (NS.EDRs). The use of NS.EDRs as a tool in applied psychophysiological research has resulted in a variety of publications. NS.EDRs are examined separately as associated with the (as a biomarker of) levels of anxiety. The aim of this study was to compare changes (in terms of amplitude, frequency and time components) in NS.EDRs at two different (pre and post of an external stimulus) resting phases. NS.EDRs (nonspecific skin conductance responses (NS.SCRs), nonspecific skin potential responses (NS.SPRs), and nonspecific skin susceptance responses (NS.SSRs)) were recorded from 50 apparently healthy volunteers simultaneously at the same skin area. They were scored as NS.SCRs and NS.SSRs for changes greater than $0.02 \mu \mathrm{S}$ and NS.SPRs greater than $0.02 \mathrm{mV}$. It was found that NS.EDRs, in particular NS.SCRs and NS.SPRs, were significantly changed in the second resting period, following the specific stimulus. More specifically, the amplitude of NS.EDRs were significantly decreased for NS.SCRs $(p<0.001)$ and for NS.SPRs $(p<0.005)$, but NS.SSRs remained stable. Moreover, the rise time of NS.SCRs was decreased in the second resting time. Furthermore, the frequency of responses was also changed. The computed NS.EDRs, in particular NS.SCRs and NS.SPRs could be of psychological interest and be used to study the electrodermal responses in detail. NS.SSRs were found to be robust with respect to nonspecific stimuli at various relaxation periods and their role was found to be less important in analysis of NS.EDRs in comparison to NS.SCRs and NS.SPRs at low frequency $(20 \mathrm{~Hz} A C$ current). This should be considered in analysis of NS.EDRs. The computed NS.EDRs, especially NS.SCRs and NS.SPRs may be used as a useful measure of arousal due to their fast response and sensitivity to nonspecific stimuli and may also be used in assessment of individual differences.
\end{abstract}

Keywords: Electrodermal activity, EDA, specific electrodermal responses, nonspecific electrodermal responses, skin conductance responses, skin potential responses

\section{Introduction}

Electrodermal activity (EDA) refers to the fluctuations in the skin electrical properties in response to sweat secretion. The term EDA was first introduced in 1966 by Johnson \& Lubin, describing all electrical phenomena in skin [1]. Sweat glands, particularly the palmar and plantar eccrine sweat glands responsible for the psychological responses, are innervated by the sympathetic nervous system, and changes in EDA are thought to reflect sympathetic nervous system arousal associated with emotion, cognition, and attention [1]. Sweating as a result of the sympathetic nervous system activation and consequently sweat glands, significantly influences the EDA responses [2], but with different characteristics.

Skin conductance responses (SCRs) increase with the sweat ducts filling and recover when sweat is reabsorbed. The same mechanisms govern the skin potential responses (SPRs), but the SPRs are more complex [3], as they change in both positive and negative directions and produce monophasic, biphasic or triphasic responses [1]. Finally skin susceptance responses (SSRs) changes are associated with the moisture content or hydration of the skin [4, 5].

The simplicity and non-invasiveness method of recordings has made EDA a common tool within various fields and attractive for several uses such as in the field of psychophysiology [6], in the field of neurology [7], measurement of arousal [8], sweating estimation [9], stress assessment $[10,11]$, assessment of psychiatric disorders $[12,13]$, pain assessment $[14,15]$, and skin moisture assessment [4].

Short-lasting variations in EDA are known as the electrodermal responses. Mainly there are two types of EDA responses, specific electrodermal responses (S.EDRs) 
and nonspecific electrodermal responses (NS.EDRs). S.EDRs are phasic changes in EDA that are associated with externally applied arousal stimuli [16]. On the other hand, NS.EDRs are responses that cannot be traced to any specific stimulation. Hence, they are called "spontaneous" or "nonspecific" responses [1].

The frequency of occurrence of NS.EDRs reflects an important psychophysiological dimension of individual differences, such as individuals showing high NS.EDRs frequency are found to differ from individuals showing low NS.EDRs frequency in EEG and behavioral measures of vigilance and attention [17, 18]. Moreover, subjects who exhibit a high NS.EDRs frequency are referred to as EDA "labiles," whereas those that exhibit low NS.EDRs frequency are considered EDA "stabiles" [19]. Furthermore, NS.EDRs are predominately common in investigations of individual differences variables such as personality $[20,21]$, health vulnerability $[22,23]$, and aggressive or antisocial behavior [24].

The NS.EDRs, particularly NS.SCRs are defined and discussed in Boucsein et al. [16], where NS.EDRs are explained as phasic increases in EDA that have the same appearance as stimulus-elicited EDA responses. However, they are considered tonic measures because they occur in the absence of external stimuli and in the absence of artifacts such as movements and sighs. NS.EDRs are usually expressed as the number of responses per unit of time (minute) and can be computed as an average over several intervals. Although, the minimum amplitudes of nonspecific SCRs is defined as 0.01 for computerized scoring and 0.05 $\mu \mathrm{S}$ for hand scoring NS.EDRs records [16], the author could not find any literature, which defines the amplitude of nonspecific SPRs and nonspecific SSRs. Therefore, all the NS.EDRs (NS.SCRs, NS.SPRs and NS.SSRs) that occurred in the absence of external stimuli and in the absence of artifacts were simultaneously recorded, even NS.SCRs with amplitude larger than the defined values.

A feature of EDA, which to the author's knowledge is not studied before, is the investigation in NS.EDRs (which occurs in the absence of external stimuli) at two different resting phases. The main aim of this study was to compare variations (in terms of amplitude, frequency and time components) in NS.EDRs at two different (pre and post of an external stimulus) resting periods through simultaneously recording the three EDA (skin conductance (SC), skin potential (SP) and skin susceptance (SS)) parameters at the same skin site.

\section{Materials and methods}

Skin complex admittance (SY) and SP were simultaneously recorded at the same electrode on the same skin site by using a PC-based EDA system. It was similar to the system presented in Tronstad et al. [9] and Bari et al. [25], consisting of a small front-end electronic box connected by means of a National Instruments DAQ card-NI USB-6211 to a PC laptop running custom-written software in LabVIEW, version 14. A three-electrode measurement setup was employed, which was composed from one measuring electrode $(M)$, one reference electrode $(R)$, and a currentsink electrode (C). The current sink electrode, together with the reference electrode, served to provide a unipolar AC SC measurement below the measuring electrode.

Simultaneously, the DC voltage between the measuring electrode and the reference electrode is utilized for SP measurement. Also a Howland current source was utilized as in Tronstad et al. [9]. A $200 \mathrm{mV}$ digital sine wave was produced via the PC, under control of LabVIEW software, converted to analog, and then fed to the Howland circuit (front-end electronic box) by the DAQ card. The Howland circuit in turn delivered a $20 \mathrm{~Hz} A C$ current of about $20 \mu \mathrm{A}$, through the measuring electrode to the skin. The DAQ card received the analog signals back from the skin through the front-end electronic box and converted it to the digital form. Then the digitized signals were processed by differentiation in the PC LabVIEW program, and separated into a DC component for SP and an AC component for SC from the real part of the SY signal and SS from the imaginary part by means of phase-sensitive rectification.

Three electrodes were placed on one arm of the test subjects at three different skin sites. The $\mathrm{M}$ electrode was placed on the hypothenar site of the palm, the R electrode on the apex of the elbow, which is an electrodermally inactive area as recommended in [26], and finally the C electrode on the underarm between $M$ and $R$. Although abrasion [27] and skin drilling [28] were recommended as a pretreatment for the inactive site, no pretreatment of the skin was used in this study to avoid any risk of contamination.

The employed electrodes were Kendall Kittycat $1050 N P S M \mathrm{Ag} / \mathrm{AgCl}$ solid gel ECG neonatal electrodes with an active electrode area of $5.05 \mathrm{~cm}^{2}$. These electrodes were chosen because they cause no or minimal wetting of the skin [29]. In addition, they are better suited than wet gel electrodes for EDA measurements [30,31], and hence are crucial for obtaining accurate EDA results.

\section{Test subjects and study protocol}

A total of 50 apparently healthy volunteers ( 25 female and 25 male) ranging in age from 18 to 66 years (mean 24.96 years) were enrolled in this study. All participants were recruited from the University of Zakho and all of them gave written informed consent before taking part in the study.

All the participants were requested to sit quietly in a chair during the experiments in a soundproof room. The room temperature was maintained $\left(22-23{ }^{\circ} \mathrm{C}\right)$, as recommended by Boucsein [1]. After a general introduction about the purpose of the experiment, the electrodes for the aim of EDA recordings were appropriately placed on one of the 
subjects' hand. Before recording, 5 min were allowed for EDA electrodes stabilization after electrode application on the participants' hands, and then NS.EDRs (NS.SCRs, NS.SPRs, and NS.SSRs) were simultaneously recorded at two different resting periods for 2 minutes ( 1 minute pre and 1 minute post a specific stimulus) as illustrated in Figure 1. In order to produce S.EDRs between the two resting periods, the participants were asked to answer a mathematical task (subtract seventeen successively from a starting number of 100) during 5 seconds. The purpose of using the external stimulus was only to separate the two resting periods and to show the pattern of NS.EDRs in pre and post of S.EDRs; therefore, all the recorded S.EDRs were ignored. Any speaking was not allowed for participants, and they were asked to relax and to avoid bodily movement, during the whole session of EDA recordings.

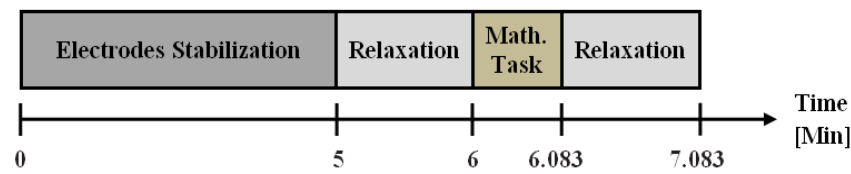

Fig.1: Time schedule of the experiment.

\section{Data analysis}

In order to analyze the difference between NS.EDRs recorded at the two resting period, that is to say before and after the math task, some scores were calculated from the EDA measurements. In order to compute these scores, the SCRs, SSRs and SPRs onsets and peaks were first specified from NS.EDRs for each subject. Locating the onsets and peaks were specified similar to the procedure presented in Bari et al. (26). All the NS.SCRs NS.SSRs greater than $0.02 \mu \mathrm{S}$ and all NS.SPRs greater than $0.02 \mathrm{mV}$ were counted and used in data analysis. Table 1 gives an illustration of the complete list of the scores used in this study.

Table 1: Overview of extracted parameters from the EDA responses that were used in the data analyses.

\begin{tabular}{ccc}
\hline Parameter & \multicolumn{1}{c}{ Description } & Unit \\
\hline NS.SCRs_Amp & $\begin{array}{c}\text { Amplitude of the nonspecific } \\
\text { skin conductance responses } \\
\text { Amplitude of the nonspecific } \\
\text { skin potential responses }\end{array}$ & $\mu \mathrm{S}$ \\
Turning point of the NS.SPRs & $\mathrm{mV}$ \\
SPRET & $\begin{array}{c}\text { Telative to the NS.SCRs peak } \\
\text { Time from onset of NS.SCRs } \\
\text { to peak NS.SCRs }\end{array}$ & $\%$ \\
\hline
\end{tabular}

Skin Potential Relative Early Turn (SPRET) was extracted for all SC and SP responses for all participants to find when the SCRs and SPRs waveforms are different. SPRET was calculated from the time of the SCRs peak minus the time of the SPRs peak, divided by the time from SCR onset to SCR peak, and multiplied by $100 \%$. This score was calculated to show the relative time difference between the turning points or peaks of the NS.SPRs and NS.SCRs [28].

\section{Statistical analysis}

The differences between NS.EDRs recorded at two relaxation periods were evaluated statistically using all the extracted scores listed in Table 1 for all test subjects. For this reason, the Wilcoxon signed-rank test and paired t-test were conducted to assess the differences, and the 0.05 level of confidence was used to define statistical significance. Moreover, in order to assess reliability among the sampled subjects (among NS.EDRs recorded over the two periods of rest), the intra-class correlation coefficient (ICC) was used. The statistical computation was performed using IBM SPSS Statistics 22.

\section{Informed consent}

Informed consent has been obtained from all individuals included in this study.

\section{Ethical approval}

The protocol has been complied with all the relevant national regulations, institutional policies and in accordance with the tenets of the Helsinki Declaration.

Results

Frequency of nonspecific electrodermal responses

Shown in Table 2 is the frequency of NS.SCRs and NS.SPRs, computed for each subject during relaxation periods (i.e. before and after the math task). It can be seen that almost all subjects gave a series of responses and there is a high variation between participants and between responses recorded at the two resting periods. Moreover, the subject number 29 showed the highest responding rate of NS.SCRs and NS.SPRs, which were 15 NS.SCRs at the first and 14 NS.SPRs at the second period, whereas the responding of subject number 34 was the lowest, with 1 NS.SCRs and 0 NS.SPRs at the first and 0 NS.SCRs and 2 NS.SPRs at the second relaxing period. The statistical analysis on the frequency of NS.SCRs and NS.SPRs yielded a non-significant $(p>0.05)$ difference between data obtained from the two different relaxation periods. Moreover, estimated ICC and their $95 \%$ confident intervals for both the frequency of NS.SCRs and NS.SPRs, are presented in Table 3. The ICC value for the frequency of NS.SCRs, was 0.773 for single measures and 0.872 for average measures. The ICC value for the frequency of NS.SPRs was also calculated, and it was 0.755 for single measures and 0.860 for average measures. This indicates that the level of reliability can be regarded as "good" to "excellent" among the sampled subjects for the frequency of both NS.SCRs and NS.SPRs.

The frequency data of NS.SCRs for the total sample are displayed in Figures $2 \mathrm{~A}$ and $2 \mathrm{~B}$, and the frequency data of NS.SPRs are shown in Figures $3 \mathrm{~A}$ and $3 \mathrm{~B}$. It can be seen through the figures that there are differences between frequencies recorded during the two resting periods. 
Table 2: Frequency of subjects NS.SCRs and NS.SPRs responding in relaxation periods

\begin{tabular}{|c|c|c|c|c|}
\hline \multirow[b]{2}{*}{ Subject } & \multicolumn{2}{|c|}{ NS.SCRs } & \multicolumn{2}{|c|}{ NS.SPRs } \\
\hline & $\begin{array}{c}1^{\text {st }} \text { period } \\
\pm 3.37\end{array}$ & $\begin{array}{c}2^{\text {nd }} \text { period } \\
\pm 3.83\end{array}$ & $\begin{array}{c}1^{\text {st }} \text { period } \\
\pm 3.34\end{array}$ & $\begin{array}{c}2^{\text {nd }} \text { period } \\
\pm 3.75\end{array}$ \\
\hline 1 & 6 & 8 & 6 & 8 \\
\hline 2 & 7 & 6 & 7 & 6 \\
\hline 3 & 10 & 15 & 9 & 14 \\
\hline 4 & 4 & 3 & 3 & 2 \\
\hline 5 & 7 & 4 & 7 & 4 \\
\hline 6 & 5 & 2 & 5 & 2 \\
\hline 7 & 9 & 10 & 9 & 10 \\
\hline 8 & 8 & 4 & 8 & 4 \\
\hline 9 & 6 & 9 & 6 & 9 \\
\hline 10 & 2 & 0 & 4 & 1 \\
\hline 11 & 11 & 6 & 11 & 6 \\
\hline 12 & 8 & 13 & 8 & 13 \\
\hline 13 & 8 & 7 & 8 & 7 \\
\hline 14 & 7 & 9 & 7 & 9 \\
\hline 15 & 7 & 2 & 7 & 2 \\
\hline 16 & 3 & 5 & 3 & 5 \\
\hline 17 & 3 & 3 & 3 & 3 \\
\hline 18 & 3 & 1 & 3 & 1 \\
\hline 19 & 3 & 2 & 3 & 2 \\
\hline 20 & 5 & 7 & 5 & 7 \\
\hline 21 & 6 & 8 & 6 & 8 \\
\hline 22 & 10 & 7 & 10 & 7 \\
\hline 23 & 3 & 1 & 3 & 1 \\
\hline 24 & 8 & 7 & 8 & 7 \\
\hline 25 & 3 & 3 & 3 & 3 \\
\hline 26 & 4 & 5 & 4 & 6 \\
\hline 27 & 11 & 9 & 11 & 9 \\
\hline 28 & 2 & 1 & 2 & 5 \\
\hline 29 & 15 & 14 & 15 & 16 \\
\hline 30 & 7 & 8 & 7 & 8 \\
\hline 31 & 10 & 8 & 10 & 8 \\
\hline 32 & 2 & 6 & 2 & 6 \\
\hline 33 & 5 & 5 & 5 & 5 \\
\hline 34 & 1 & 0 & 0 & 2 \\
\hline 35 & 14 & 17 & 14 & 17 \\
\hline 36 & 2 & 6 & 2 & 6 \\
\hline 37 & 5 & 2 & 5 & 2 \\
\hline 38 & 11 & 10 & 11 & 10 \\
\hline 39 & 5 & 6 & 5 & 6 \\
\hline 40 & 8 & 6 & 8 & 6 \\
\hline 41 & 7 & 5 & 7 & 5 \\
\hline 42 & 2 & 5 & 3 & 5 \\
\hline 43 & 2 & 1 & 2 & 1 \\
\hline 44 & 4 & 8 & 4 & 8 \\
\hline 45 & 3 & 5 & 3 & 5 \\
\hline 46 & 5 & 5 & 5 & 5 \\
\hline 47 & 6 & 5 & 6 & 5 \\
\hline 48 & 8 & 8 & 8 & 8 \\
\hline 49 & 11 & 11 & 11 & 11 \\
\hline 50 & 1 & 2 & 1 & 2 \\
\hline
\end{tabular}

Table 3: Intraclass correlation coefficient (ICC) for the frequency of NS. SCRs and NS.SPRs obtained from the two relaxation periods

\begin{tabular}{lccc}
\hline \multirow{2}{*}{ Parameters } & $\begin{array}{l}\text { Intraclass } \\
\text { correlation }\end{array}$ & Lower & Up\% Cl \\
\hline NS.SCRs & $0.773^{\mathrm{a}}$ & 0.632 & 0.865 \\
& $0.872^{\mathrm{b}}$ & 0.774 & 0.928 \\
NS.SPRs & $0.755^{\mathrm{a}}$ & 0.604 & 0.853 \\
& $0.860^{\mathrm{b}}$ & 0.753 & 0.921 \\
\hline
\end{tabular}

$a=$ single measures, $b=$ average measures

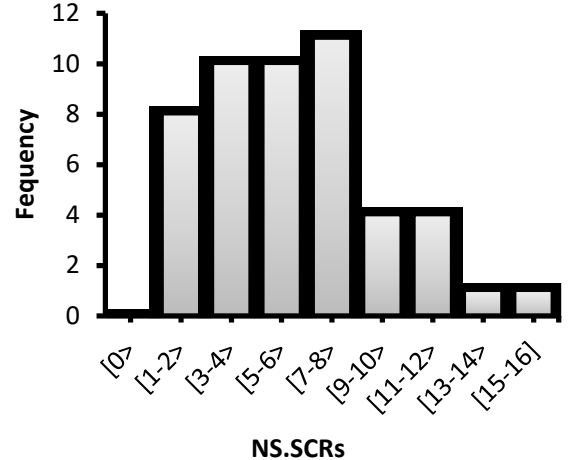

(A)

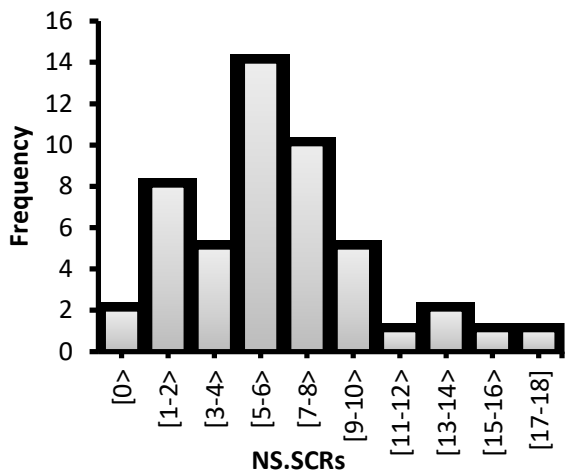

(B)

Fig. 2: Distribution of NS.SCRs frequency for the total sample $(n=50)$ : (A) first resting period, and (B) second resting period.

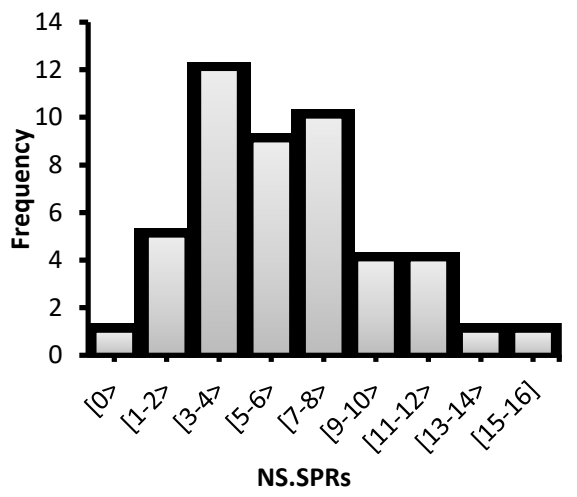

(A)

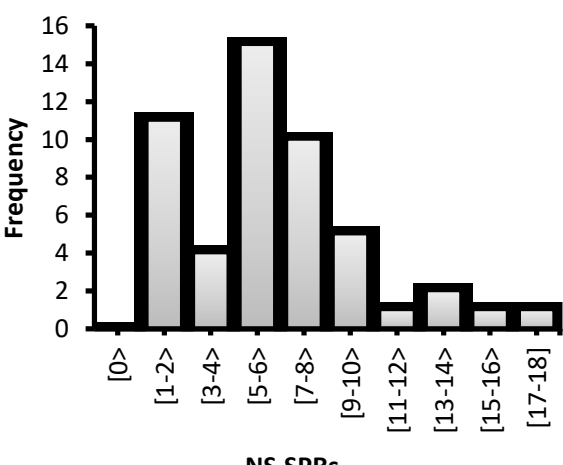

(B)

Fig. 3: Distribution of NS.SPRs-frequency for the total sample $(n=50)$ : (A) first resting period, and (B) second resting period. 


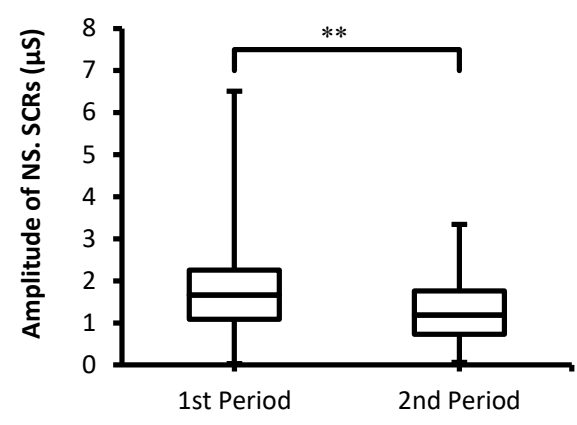

Average of NS.SCRs

Fig. 4: Box-plot with medians, quartiles and the min and max as whiskers shows average value of NS.SCRs_Amp at relaxation periods for all participants $(\mathrm{n}=50) .{ }^{* *} p<0.001$

Moreover, frequency of most NS.SCRs at the first period was from 1 to 8 , but this trend was changed in the second period (after the math task) and the frequency was between 5 and 8 responses. The frequency of NS.SPRs was also changed, and it was between 3 and 8 in the first and 12 and 5-8 in the second resting period.

\section{Amplitude of response}

\section{Skin conductance response}

The data for mean amplitude of NS.SCRs are presented in Figure 4. It can be seen that the median amplitude of NS.SCRs that come at the first relaxing time (before the math task) is larger than the median amplitude of NS.SCRs that come at the second relaxing time. Moreover, once the statistical tests were performed, a significant $(p<0.001)$ difference was obtained.

\section{Skin potential response}

When data for NS.SPRs mean amplitude were analyzed, a significant $(p<0.005)$ difference was found. Figure 5 shows mean amplitude of NS.SPRs. A similar pattern of results was obtained for NS.SPRs and the median amplitude of NS.SPRs is decreased following the math task, in the second resting period.

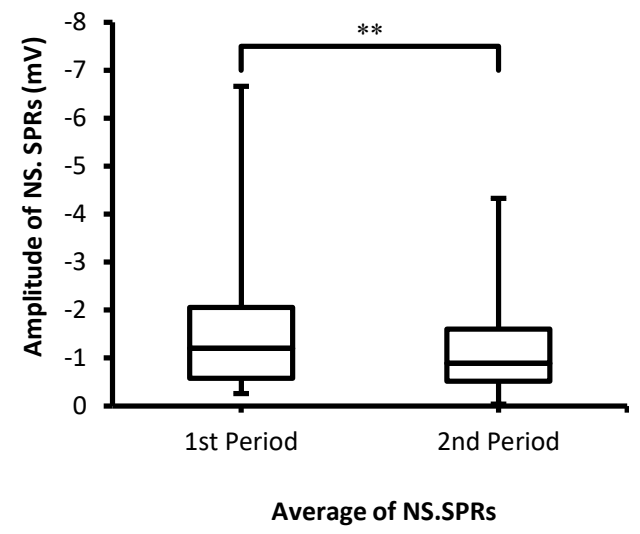

Fig. 5: Box-plot with medians, quartiles and the min and max as whiskers shows average value of NS.SPRs_Amp at relaxation periods for all participants $(n=50) .{ }^{* *} p<0.005$

\section{Times of components of response}

\section{Rise time of NS.SCRS}

Statistical analysis on rise time data showed a significant difference $(p<0.005)$ between rise time of NS.SCRs recorded at two resting times. In addition, it is clear from Figure 6 that, the median rise time value of NS.SCRs is decreased in the second relaxation period, following the math task.

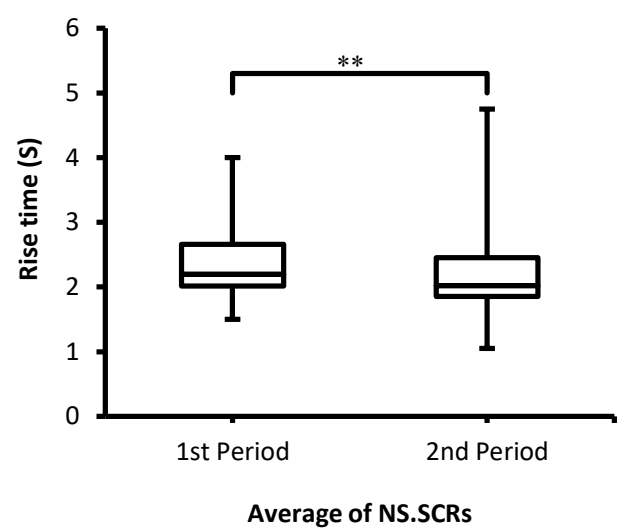

Fig. 6: Box-plot with medians, quartiles and the min and max as whiskers shows average value of NS.SCRs_Tris at relaxation periods for all participants $(\mathrm{n}=50) .{ }^{* *} p<0.005$

\section{Skin Potential Relative Early Turn (SPRET)}

Box plot (Figure 7) comparing percentage of mean SPRET for all participants, shows significant difference in SPRET computed from NS.SCRs and NS.SPRs at relaxation periods. It is clear from the plot that the median SPRET value is significantly decreased in the second resting period. Statistical analysis also yielded a significant $(p<0.001)$ difference between data from different relaxation periods.

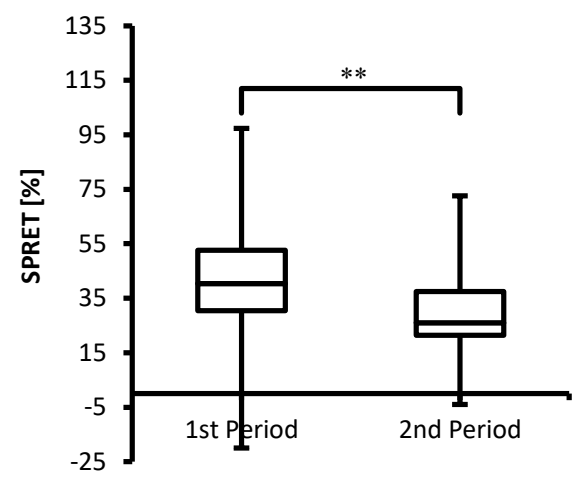

Average NS. SPRET

Fig. 7: Box-plot with medians, quartiles and the min and max as whiskers shows average value of SPRET at relaxation periods for all participants $(n=50) . * * p<0.001$

NS.SSRs recorded from all the participants were very small (smaller than $0.01 \mu \mathrm{S}$ ) or there were no responses at all. Figure 8 shows a typical EDA recording from a participant. It is clear from the figure that at all the instance of the NS.SCRs and NS.SPRs, there were no NS.SSRs; however, at the time of introducing the specific stimulus, the three EDA (SCRs, SPRs and SSRs) parameters simultaneously respond to the stimulus. 


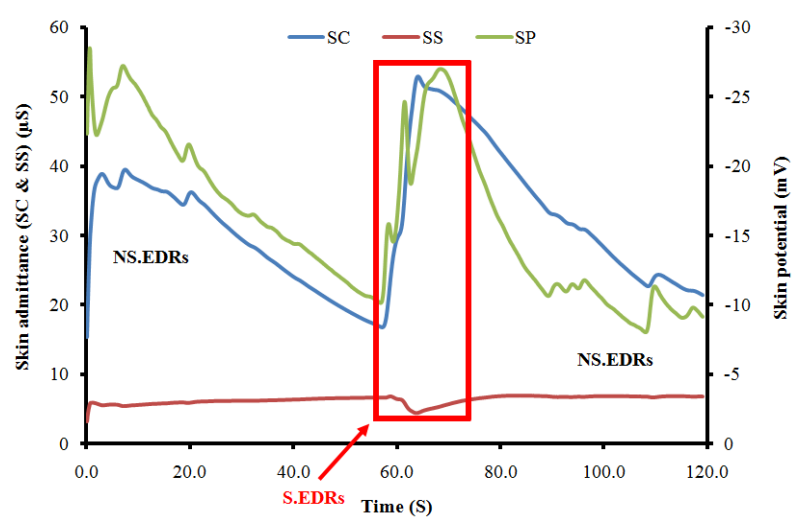

Fig. 8: SCRs, SSRs, and SPRs recorded from a test subjects, demonstrating stable waveform of SSRs in response to nonspecific stimuli.

\section{Discussion}

The purpose of the present study was to examine nonspecific electrodermal responses (NS.EDRs) at two different relaxation periods (pre and post of a specific stimulus) and find out whether there are any difference (in terms of amplitude, frequency and time components) between NS.EDRs that were recorded post of the stimulus compared to those measured pre of the stimulus, through simultaneously recording the three EDA (SC, SP and SS) parameters.

The study results suggest the possibility of changes in frequency of responses, time components and amplitude of NS.EDRs measured in the second resting period (post of the math task (stimulus)) in contrast to NS.EDRs recorded in the first period. However, there was great disparity among the test subjects according to the data seen in Tables 2, which is due to individual differences (labiles and stabiles).

Moreover, Nikula et al. [32], indicated that the observed NS.EDRs during resting conditions are connected with conscious rumination on motivationally relevant but unrealized goals and intentions. Since such rumination could be considered an effortful process, NS.EDRs may reflect arousal in the service of increased cognitive capacity during rumination. Therefore, stable and consistent individual differences in resting NS.EDRs lability may index differential propensities toward effortful ideation [33]. NS.EDRs lability is certainly associated with self-reports of emotional ideation during relaxation conditions [34] and with observer ratings of active suppression of negative thoughts while anticipating an aversive stimulus [35].

It should furthermore be noted that the frequency of NS.EDRs (both NS.SCRs and NS.SPRs) was changed in the second resting period, following the math task as indicated by Figures 2 and 3 . This means that there should be a psychological reason, which causes these changes; although, such changes were statistically insignificant ( $p>0.05$ ). In addition, the analysis with ICC (Table 3 ) showed that the level of reliability, which reflects both degree of correlation and agreement between measurements of both
NS.SCRs and NS.SPRs obtained from the two resting periods is from "good" to "excellent". According to Bernstein [37] the status of a subject in terms of NS.EDRs frequency depends on the conditions or occasion under which the frequency is obtained, then the interaction of subjects and conditions or subjects and sessions can be major contributors to changes in NS.EDRs frequency, and this frequency may reflects a trait-like characteristic of subjects.

The results for mean NS.SCRs amplitude, which allows for determining the total amount of nonspecific changes in EDA [1], presented in Figure 4, indicate that there is a significant $(p<0.001)$ difference between mean amplitude of NS.SCRs measured in the first and second resting periods. In addition, the median values of NS.SCRs amplitudes were decreased in the second relaxation period after the math task. This reduction trend may be due to habituation in the individuals' reactions to external stimuli, leading to a decrease in the amplitude of the NS.SCRs. Furthermore, this also may be due to change or decrease in the level of stress response of individuals with the passage of time, since EDA responses are strongly associated with mental stress [37]. The same results was also found with simultaneously measured NS.SPRs (Figure 5), in which the amplitude of NS.SPRs is significantly $(p<0.005)$ decreased in the second resting period.

Data of the present study also point to changes in time dependent EDA scores. Figure 6 shows rise time of NS.SCRs. It can be seen that, rise time of the responses is decreased in the second resting time following the math task. This suggests that, the shorter rise time is associated with the weaker NS.SCRs [25]. Figure 7 indicates a significant $(p<0.001)$ reduction in mean SPRET value in the second period, although more negative SPRET values are obtained in the first period. This means that in the first period, some of the NS.SPRs peak at a later time than the NS.SCRs peak (negative SPRET) [29], whereas the negative SPRET values is reduced in the second period, which mean that the NS.SPRs peaked earlier than the NS.SCRs peak.

The study results (see Figure 8 for example) show that SS is a more robust parameter than SC and SP in response to nonspecific stimuli, since no NS.SSRs greater than 0.02 $\mu \mathrm{S}$ were detected in any participant, neither in the first nor second resting periods. This means that SSRs are not influenced by nonspecific stimuli. Moreover, the nonspecific stimuli may not lead to changes in skin hydration as a result of sweat secretion and then cause changes in SS (as noted in this paper, SSRs changes are associated with the moisture content or hydration of the skin), because these stimuli are very fast and short and perhaps there is not enough time for skin to undergo such changes.

\section{Conclusion}

The subjects' NS.EDRs were rated to be significantly more intense at the first resting period (pre of the specific 
stimulus) of the experiment compared to the NS.EDRs recorded in the second resting periods (post of the specific stimulus) as indicated by NS.SCRs and NS.SPRs. Moreover, the NS.EDRs that were measured at the second resting period, were different in frequency of responses and time components and of smaller amplitude compared to those detected at the first resting period. NS.SSRs recorded from all the participants were very small or even not detectable, and found to be robust with respect to nonspecific stimuli. This suggests that, the role of SS is less important in analysis of NS.EDRs with respect to the SC and SP at low frequency (20 Hz AC current). This is important to consider in the recording or analysis of NS.EDRs.

The computed NS.EDRs, in particular NS.SCRs and NS.SPRs could be of physiological interest and be used to study the electrodermal responses in detail. In addition, such responses may be used as a useful measure of arousal and in assessing individual differences in feelings of excitement or stress under various conditions due to their fast response and sensitivity.

\section{Conflict of interest}

Author states no conflict of interest.

\section{References}

1. Boucsein W. Electrodermal activity. Berlin: Plenum Press; 2012.

2. Grimnes $S$, Martinsen $\varnothing \mathrm{G}$. Bioimpedance and Bioelectricity Basics. 3rd ed. Oxford: Elsevier; 2015. https://doi.org/10.1016/b978-0-12-411470-8.00011-8

3. Edelberg R. Electrodermal mechanisms: A critique of the twoeffector hypothesis and a proposed replacement. In: Roy JC, Boucsein W, Fowles DC, Gruzelier JH, editors. Progress in Electrodermal Research. New York: Plenum Press; 1993. p. 730. https://doi.org/10.1007/978-1-4615-2864-7_2

4. Martinsen $\varnothing \mathrm{G}$, Grimnes $S$, Karlsen J. Electrical methods for skin moisture assessment. Skin Pharmacol Physio.1995; 8: 237-45. https://doi.org/10.1159/000211353

5. Martinsen $\varnothing \mathrm{G}$, Grimnes $S$, Nilsen JK, Tronstad C, Jang W, Kim $\mathrm{H}$, et al. Gravimetric method for in vitro calibration of skin hydration measurements. IEEE Trans Biomed Eng. 2008; 55:728-32. https://doi.org/10.1109/tbme.2007.912651

6. Martin I, Venables PH. Techniques in psychophysiology. Chichester: Wiley \& Sons; 1980.

7. Kucera P, Goldenberg Z, Kurca E. Sympathetic skin response: review of the method and its clinical use. Bratisl Med J. 2004; 105:108-16.

8. Poh M-Z, Swenson NC, Picard RW. A wearable sensor for unobtrusive, long-term assessment of electrodermal activity. IEEE T Biomed Eng. 2010;57(5):1243-52. https://doi.org/10.1109/tbme.2009.2038487

9. Tronstad C, Kalvøy H, Grimnes S, Martinsen $\varnothing \mathrm{G}$. Improved estimation of sweating based on electrical properties of skin. Annals Biomed Eng. 2013; 41:1074-83. https://doi.org/10.1007/s10439-013-0743-4
10. Setz C, Arnrich B, Schumm J, La Marca R, Tröster G, Ehlert U. Discriminating stress from cognitive load using a wearable EDA device. IEEE T Inf Technol B. 2009;14(2):410-7. https://doi.org/10.1109/titb.2009.2036164

11. Healey JA, Picard RW. Detecting stress during real-world driving tasks using physiological sensors. IEEE Trans Intell Transp Syst. 2005; 6:156-66. https://doi.org/10.1109/tits.2005.848368

12. Nilsson BM, Hultman CM, Wiesel F-A. Niacin skin-flush response and electrodermal activity in patients with schizophrenia and healthy controls. Prostag Leukotr Ess. 2006;74(5):339-46. https://doi.org/10.1016/j.plefa.2006.02.002

13. lacono WG, Carlson SR, Taylor J, Elkins IJ, McGue M. Behavioral disinhibition and the development of substanceuse disorders: findings from the Minnesota Twin Family Study. Dev Psychopathol. 1999;11(4):869-900. https://doi.org/10.1017/s0954579499002369

14. Hullett B, Chambers N, Preuss J, Zamudio I, Lange J, Pascoe E, et al. Monitoring Electrical Skin ConductanceA Tool for the Assessment of Postoperative Pain in Children? Anesthesiology. 2009;111(3):513-517. https://doi.org/10.1097/aln.0b013e3181b27c18

15. Bari DS, Aldosky HYY, Tronstad C, Kalvøy H, Martinsen $\varnothing \mathrm{G}$. Electrodermal activity responses for quantitative assessment of felt pain. J Electr Bioimp. 2018;9(1):52-8. https://doi.org/10.2478/joeb-2018-0010

16. Boucsein W, Fowles DC, Grimnes S, Ben-Shakhar G, Roth WT, Dawson ME, et al. Publication recommendations for electrodermal measurements. Psychophysiology. 2012;49(8):1017-34.

17. Bohlin G. Interaction of arousal and habituation in the development of sleep during monotonous stimulation. Biol Psychol. 1973;1(2):99-114. https://doi.org/10.1016/0301-0511(73)90002-1

18. Katkin ES. Electrodermal lability: A psychophysiological analysis of individual differences in response to stress. In: Sarason IG, Spielberger CD, editors. Stress and anxiety. New York: Wiley; 1975. p.141-176.

19. Crider A, Lunn R. Electrodermal lability as a personality dimension. J Exp Res Pers. 1971;5(2):145-150.

20. Crider A. Personality and electrodermal response lability: An interpretation. Appl Psychophysiol Biofeedback. 2008;33(3):141. https://doi.org/10.1007/s10484-008-9057-y

21. Norris CJ, Larsen JT, Cacioppo JT. Neuroticism is associated with larger and more prolonged electrodermal responses to emotionally evocative pictures. Psychophysiology. 2007;44(5):823-6. https://doi.org/10.1111/j.1469-8986.2007.00551.x

22. El-Sheikh M, Arsiwalla DD. Children's sleep, skin conductance level and mental health. J Sleep Res. 2011;20(2):326-37. https://doi.org/10.1111/j.1365-2869.2010.00880.x

23. El-Sheikh M, Keller PS, Erath SA. Marital conflict and risk for child maladjustment over time: Skin conductance level reactivity as a vulnerability factor. J Abnorm Child Psychol. 2007;35(5):715-27. https://doi.org/10.1007/s10802-007-9127-2 
24. Gatzke-Kopp LM, Raine A, Loeber R, Stouthamer-Loeber M, Steinhauer SR. Serious delinquent behavior, sensation seeking, and electrodermal arousal. J Abnorm Child Psychol. 2002;30(5):477-86.

https://doi.org/10.1023/a:1019816930615

25. Bari DS, Aldosky HYY, Tronstad C, Kalvøy H, Martinsen $\varnothing \mathrm{G}$. Electrodermal responses to discrete stimuli measured by skin conductance, skin potential, and skin susceptance. Skin Research and Technology. 2018;24(1):108-16. https://doi.org/10.1111/srt.12397

26. Fowles DC, Christie MJ, Edelberg R, Grings WW, Lykken DT, Venables PH. Publication recommendations for electrodermal measurements. Psychophysiology. 1981;18(3):232-9. https://doi.org/10.1111/j.1469-8986.1981.tb03024.x

27. Edelberg R. Electrical properties of the skin. In: Brown CC, editors. Methods in psychophysiology. Baltimore: Williams \& Wilkins; 1967. p. 1-53.

28. Venables PH, Christie MJ. Electrodermal activity. In: Martin I, Venables $\mathrm{PH}$, editors. Techniques in psychophysiology. New York: Wiley; 1980. p. 3-67.

29. Tronstad C, Kalvøy H, Grimnes S, Martinsen $\varnothing \mathrm{G}$. Waveform difference between skin conductance and skin potential responses in relation to electrical and evaporative properties of skin. Psychophysiology. 2013; 50:1070-78. https://doi.org/10.1111/psyp.12092

30. Tronstad C, Johnsen GK, Grimnes S, Martinsen $\varnothing \mathrm{G}$. A study on electrode gels for skin conductance measurements. Physiol Meas. 2010; 31:1395-410. https://doi.org/10.1088/0967-3334/31/10/008
31. Martinsen $\varnothing \mathrm{G}$, Pabst O,Tronstad C, Grimnes S. Sources of error in AC measurement of skin conductance. J Electr Bioimp. 2015; 6: 49-53. https://doi.org/10.5617/jeb.2640

32. Nikula R, Klinger E, Larson-Gutman MK. Current concerns and electrodermal reactivity: Responses to words and thoughts. J Pers. 1993;61(1):63-84. https://doi.org/10.1111/j.1467-6494.1993.tb00279.x

33. Crider A, Kremen WS, Xian H, Jacobson KC, Waterman B, Eisen SA, et al. Stability, consistency, and heritability of electrodermal response lability in middle-aged male twins. Psychophysiology. 2004;41(4):501-9. https://doi.org/10.1111/j.1469-8986.2004.00189.x

34. Baugher DM. An examination of the nonspecific skin resistance response. Bull Psychon Soc. 1975;6(3):254-6. https://doi.org/10.3758/bf03336654

35. Hare RD. Denial of threat and emotional response to impending painful stimulation. J Consult Psychol. 1966;30(4):359. https://doi.org/10.1037/h0023547

36. Bernstein AS. The orienting response as novelty and significance detector: Reply to O'Gorman. Psychophysiology. 1979;16(3):263-73. https://doi.org/10.1111/j.1469-8986.1979.tb02989.x

37. Bach DR, Flandin G, Friston KJ, Dolan RJ. Time-series analysis for rapid event-related skin conductance responses. J Neurosci Methods. 2009;184(2):224-34. https://doi.org/10.1016/j.jneumeth.2009.08.005 